\title{
MATERIALIEN
}

WIENER SLAVISTISCHES JAHRBUCH, Band 54/2008, 245-254

(C) 2008 by Österreichische Akademie der Wissenschaften, Wien

Das Institut für Slawistik der Universität Wien und die Redaktion des Wiener Slavistischen Jahrbuchs gratulieren Heinz Miklas zum 60. Geburtstag.

\section{Schriftenverzeichnis von Heinz Miklas (Stand: März 2008)}

A. Monographien und sonstige Buchpublikationen:

1. Die Dioptra des Philippos Monotropos im Slavischen. Allgemeine Untersuchung und Text des ersten Buches. Dissertation: Graz, im April 1975 (CVI. 96 Doppels. - Offset-Druck, 22 Expl.).

2. Festschrift für Wilhelm Lettenbauer zum 70. Geburtstag, herausgegeben von Antonín Měšt'an und Eckhard Weiher unter Mitarbeit von Peter Drews und Heinz Miklas. Freiburg i. Br.: U. W. Weiher, 1982 (298 S.).

3. Die Dogmatik des Johannes von Damaskus in der kirchenslavischen Übersetzung des 14. Jahrhunderts, herausgegeben von Eckhard Weiher unter Mitarbeit von Felix Keller und Heinz Miklas (Die Dogmatik ... in den slavischen Übersetzungen des 14. bis 18. Jahrhunderts, herausgegeben von Eckhard Weiher, Bd. 1) (Monumenta linguae slavicae dialecti veteris 25). Freiburg i. Br.: U.W. Weiher, 1987 (LX.816 S. u. 23 Tafeln im Großformat).

4. Berlinski Sbornik. Vollständige Studienausgabe im Originalformat von Ms. (slav.) Wuk 48 aus dem Besitz der Staatsbibliothek Preußischer Kulturbesitz, Berlin, und von Ms. 0. p. I.15 der Staatlichen Öffentlichen Bibliothek ,M. E. Saltykov-Ščedrin “, Leningrad. Eingeleitet und herausgegeben mit Ergänzungen aus weiteren Quellen von Heinz Miklas. Mit einem Anhang von Vjačeslav M. Zagrebin (Codices selecti 79). Graz: Akademische Druck- u. Verlagsanstalt, 1988 (115 S. Einleitung u. 367 Texts. in Reprographie). 
5. Vorläufiger Katalog kirchenslavischer Homilien des beweglichen Jahreszyklus aus Handschriften des 11.-16. Jahrhunderts vorwiegend ostslavischer Provenienz. Zusammengestellt von Tat'jana V. Čertorickaja unter der Redaktion von Heinz Miklas / Predvaritel'nyj katalog cerkovnoslavjanskich gomilij podvižnogo kalendarnogo cikla po rukopisjam XI-XVI vv. preimuščestvenno vostočnoslavjanskogo proischoždenija. Sostavlen Tat'janoj V. Čertorickoj pod redakciej Chajnc Miklas (Nordrh.-Westf. AdW. Patristica Slavica 1). Opladen: Westdeutscher Verlag, 1994 (795 S.).

6. (mit Božidar Rajkov, Stefan Kožucharov u. Christo Kodov) Katalog na slavjanskite răkopisi v bibliotekata na Zografskija manastir v Sveta gora / Katalog der slavischen Handschriften im Athoskloster Zographou, Heiliger Berg. Sofija: CIBAL, 1994 (158 S. u. 285 Tafeln).

7. Die Dogmatik des Johannes von Damaskus in der Übersetzung des Fürsten Andrej M. Kurbskij (1528-1583), herausgegeben von Juliane Besters-Dilger unter Mitarbeit von Eckhard Weiher, Felix Keller und Heinz Miklas (MLS 35). Freiburg i. Br.: U. W. Weiher, 1995 (928 S. u. 26 Tafeln im Großformat).

8. Psalterii Sinaitici pars nova (monasterii s. Catharinae codex slav. 2/N). Ad editionem praeparaverunt Petra Fetková, Zoe Hauptová, Václav Konzal, Ludmila Pacnerová, Jana Švábová; sub redactione Francisci V. Mareš † (Heinz Miklas). (ÖAW, Phil.-hist. Kl., Schriften der Balkan-Kommission, Philologische Abt. 38, Fontes Nr. 2). Wien: ÖAW, 1997 (XXIII. 201 S.).

9. Das Tetraevangelium des Makarije aus dem Jahre 1512. Der erste kirchenslavische Evangeliendruck. Faksimile-Ausgabe, besorgt und eingeleitet von Heinz Miklas unter Mitwirkung von Ştefan Godorogea und Christian Hannick (Biblia slavica, Serie IV: Südslavische Bibeln, Band 1). Paderborn/München etc.: Ferdinand Schöningh, 1999 (XLVIII S. u. 291 Tafeln).

10. Slawistik an der Universität Wien 1849-1999, ed. Institut für Slawistik der Univ. Wien unter der Redaktion von Juliane Besters-Dilger und Heinz Miklas. Wien 1999 (50 S.).

11. Glagolitica - Zum Ursprung der slavischen Schriftkultur (ÖAW, Phil.-hist. K1., Schriften der Balkan-Kommission, Philologische Abt. 41), herausgegeben von H. Miklas unter der Mitarbeit von Sylvia Richter und Velizar Sadovski. Wien: ÖAW, 2000 (260 S.).

12. Nikolaj S. Trubetzkoy, Russland - Europa - Eurasien. Ausgewählte Schriften zur Kulturwissenschaft. Herausgegeben von Fedor B. Poljakov. Redaktion und Vorwort Heinz Miklas (ÖAW. Phil.-hist. K1., Schriften der Balkan-Kommission 45). Wien: ÖAW, 2005 (479 S.).

13. Contribution à l'histoire de la slavistique dans les pays non slaves / Beiträge zur Geschichte der Slawistik in den nichtslawischen Ländern / K istorii slavistiki v neslavjanskich stranach, edd. Giovanna Brogi Bercoff, Pierre Gonneau, Heinz Miklas (ÖAW. Phil.-hist. Kl. Schriften der Balkan-Kommission 46 / Institut 
d'études slaves, Traveaux publiés par l'Institut d'études slaves XLVI). Wien: ÖAW, 2005 (572 S.).

14. Wort - Geist - Kultur. Gedenkschrift für Sergej S. Averincev, herausgegeben von Juliane Besters-Dilger, Heinz Miklas, Gerhard Neweklowsky und Fedor B. Poljakov (Russkaja kul'tura v Evrope 2). Frankfurt a. M./Berlin etc.: Peter Lang, 2006 (493 S.).

15. Berlinski Sbornik. Srednobălgarski pametnik ot načaloto na XIV vek, s dopălnenija ot drugi răkopisi. Izdanieto e podgotveno to Chajnc Miklas, Lora Taseva, Marija Jovčeva / Berlinski Sbornik. Ein kirchenslavisches Denkmal mittelbulgarischer Redaktion des beginnenden 14. Jahrhunderts, ergänzt aus weiteren handschriftlichen Quellen, herausgegeben von Heinz Miklas, Lora Taseva, Marija Jovčeva (ÖAW, Phil.-hist. K1., Schriften der Balkan-Kommission, Bd. 47, Fontes Nr. 3). Sofia: BAN, Kyrillomethodianisches Forschungszentrum / Wien: ÖAW, 2006 (457 S.).

B. Aufsätze und Rezensionsartikel:

1. Ein Beitrag zu den slavischen Handschriften auf dem Athos, in: Palaeobulgarica I (Sofia 1977) 1, 65-75.

2. Kăm văprosa za slavjanskija prevod na Filipovoto săčinenie Dioptra, in: Starobălgarska literatura. Izsledvanija i materiali 2 (Sofija 1977), 169-181.

3. Pogled vărchu Filipovata "Dioptra", in: Starobălgarska literatura 3 (Sofija 1978), 56-61.

4. Zwei russische liturgische Gebrauchskodizes aus privatem Besitz, in: Die Welt der Slaven XXIV.1 (N.F. III.1) (München 1979), 185-190.

5. Ein interessanter Neuerwerb der Universitätsbibliothek Freiburg i. Br., in: Die Welt der Slaven XXIV.2 (N.F. III.2) (München 1979), 298-307.

6. Ergebnisse und Perspektiven bei der Erforschung der kirchenslavisch-griechischen Übersetzungstheorie und Übersetzungspraxis. - Klaus TROST, Untersuchungen zur Übersetzungstheorie und -praxis des späteren Kirchenslavischen. Die Abstrakta in der Hexaemeronübersetzung des Zagreber Zbornik von 1469. München: W. Fink, 1978. 381 S. (Forum slavicum 43), in: Palaeobulgarica IV (Sofia 1980) 3, 98-111.

7. Zur kirchenslavischen Überlieferung der Häresiengeschichte des Johannes von Damaskus, in: FS für Linda Sadnik zum 70. Geburtstag, herausgegeben von Eckhard Weiher (MLS 15). Freiburg i. Br. 1981, 323-387.

8. Zur Edition der Dioptra des Philippos Monotropos, in: XVI. Int. Byzantinistenkongreß. Akten I. Hauptreferate, Beiheft (Jahrbuch d. Österr. Byzantinistik 31/ Beiheft). Wien 1981, sub 2.1 (5 S.).

9. Tradition und Innovation in der bulgarischen Hochsprache des 14.-15. Jahrhunderts. - Zur bisherigen Literatur über die Sprache des Patriarchen Evtimij, insbesondere: Horst KÄMMERER, Untersuchungen zur Flexion der Substantive in der 
bulgarischen Schriftsprache des 14./15. Jahrhunderts. Ein Beitrag zur historischen Morphologie (Universität Regensburg, Slavistische Arbeiten 3). München: Trofenik, 1977, in: Anzeiger für slavische Philologie 13 (Graz 1982), 4576 .

10. Trubetzkoys Sprachbundbegriff aus der Sicht der jüngeren Forschung, in: FS für Wilhelm Lettenbauer zum 70. Geburtstag, herausgegeben von A. Měšt'an und E. Weiher unter Mitarbeit von P. Drews und H. Miklas. Freiburg i. Br. 1982, 131153.

11. Einige Bemerkungen zum sogenannten „Berlinski Sbornik“, in: Die slawischen Sprachen 1 (Salzburg 1982), 39-41.

12. Svoj-si, sebe si und Verwandtes, in: Părvi Meždunaroden kongres po bălgaristika. Sofija 23 maj- 3 juni 1981. Dokladi istoričeski razvoj na bălgarskija ezik. Sravnitelno ezikoznanie, dialektologija, prevod. Sofija 1983, 82-96.

13. (mit Jaroslav N. Ščapov und William R. Veder) The Slavic Manuscripts in the Federal Republic of Germany, in: Polata knigopisnaja 9 (Nijmegen 1984), 3958.

14. Der Nazarether Metropolit Gabriel und seine russische Übersetzung der mit dem Namen Patr. Gennadios II. verknüpften Orakeldeutung über das Schicksal Konstantinopels, in: Cyrillomethodianum 8-9 (Thessalonique 1985), 121-148.

15. Neskol'ko rezul'tatov i zadač izučenija balkanoslavjanskich narrativnych pamjatnikov (Rezjume), in: CIBAL Bulletin d'information 9 (Sofia 1985), 88-89.

16. (mit Eckhard Weiher) Prevodačeski pochvati v Bogoslovieto na Joan Ekzarch v sravnenie s pokăsni paralelni prevodi, in: Starobălgarska literatura 19 (Sofija 1986), 29-59.

17. Zur Struktur des kyrillisch-altkirchenslavischen (altbulgarischen) Schriftsystems. Ein Systematisierungsversuch, in: Palaeobulgarica XII (Sofia 1988) 3, 52-65.

18. Kyrillomethodianisches und nachkyrillomethodianisches Erbe im ersten ostslavischen Einfluß auf die südslavische Literatur, in: Symposium Methodianum. Beiträge der Int. Tagung in Regensburg (17. bis 24. April 1985) zum Gedenken an den 1100. Todestag des heiligen Method, herausgegeben von K. Trost, E. Völkl u. E. Wedel (Selecta slavica 13). Neuried 1988, 437-472.

19. CIP und ISBN-Codes zur Erfassung von Manuskripten?, in: Polata knigopisnaja 17-18 (Amsterdam 1987) 1988, 85-87.

20. Eine athonitische gottesdienstliche Sammelhandschrift in Basel, in: Arheografski prilozi 9 (Beograd 1987) 1988, 125-146.

21. Slavische Sprachreformatoren in West und Ost: Der Fall des Tschechen Jan Hus und des Bulgaren in serbischen Diensten Konstantin von Kostenec, in: Die Welt der Slaven XXXIV.1 (N.F. XIII.1) (München 1989), 18-31.

22. Paläographische und graphematische Aspekte der kyrillischen Schriftentwicklung in Bulgarien (bis zum 14. Jahrhundert), in: Kulturelle Traditionen in Bulga- 
rien, herausgegeben von R. Lauer und P. Schreiner (Abhandlungen d. AdW in Göttingen, phil.-hist. Kl. Dritte Folge, Bd. 77). Göttingen 1989, 68-90.

23. Litterae palaeoslovenice, in: Saeculum 40 (1989) 3/4 (Freiburg i. Br. 1990), 253-272.

24. Materialien zur Erforschung des Werkes Methods und seiner Schüler, in: Leben und Werk der byzantinischen Slavenapostel Methodios und Kyrillos. Beiträge eines Symposiums der Griechisch-deutschen Initiative Würzburg im Wasserschloß Mitwitz vom 25.-27. Juli 1985 zum Gedenken an den 1100. Todestag des hl. Methodios, herausgegeben von Evangelos Konstantinou. Münsterschwarzach 1991, 65-81.

25. Johannes von Kiew, in: Biographisch-bibliographisches Kirchenlexikon, begründet $\mathrm{u}$. herausgegeben von F. W. Bautz, fortgeführt von Traugott Bautz, Bd. 3, Herzberg 1992, 436-439 (http://www.bautz.de/bbkl/j/Johannes_v_kie.shtml.)

26. Klemens von Achrida (Kliment v. Ochrid), in: ibid., Bd. 4, 15-24 (http://www. bautz.de/bbkl/k/Klemens_v_a.shtml.)

27. Ot Preslavskija săbor do Preslavskata škola. Văprosi na grafematikata, in: Palaeobulgarica XVII (Sofia 1993) 3, 3-12.

28. Möglichkeiten der Textentwicklung bei prolongierter Überlieferung in der Tradition der Slavia orthodoxa, in: H.L.C. Tristram (Hrsg.): Text und Zeittiefe. (Script-Oralia 58). Tübingen 1993, 43-69.

29. (mit Maria Schnitter) Kyrillomethodianische Miszellen, in: Anzeiger für Slavische Philologie XXII (1994) 1, 141-220.

30. Kăde să otišli parorijskite răkopisi?, in: G. Dančev et al. (Hrsg.), Pametnici, poetika, istoriografija. Peti meždunar. simpozium Veliko Tărnovo, 6-8 septemvri 1989g. (Tărnovska knižovna škola 5), Veliko Tărnovo 1994, 29-43.

31. Zur Typologie der altkyrillischen kürzenden Graphie, in: Polata knigopisnaja 27-28 (Amsterdam, Dec. 1995), 37-60.

32. Johannes II. (Ioann), Metr. v. Kiev, in: Lexikon für Theologie und Kirche, Bd. 6. Freiburg: Herder, 1996, 925.

33. Griechisches Schriftdenken und slavische Schriftlichkeit: Glagolica und Kyrillica zwischen Verschriftung und Verschriftlichung, in: Das slawische Phänomen. FS für Prof. Dr. Antonín Měšt'an zu seinem 65. Geburtstag, herausgegeben von Karel Mácha. Prag 1996, 117-124.

34. Kliment v. Ochrid/Konstantin v. Kostenec/Konstantin v. Preslav/Kosmas (Kozma Presviter), in: Lexikon für Theologie und Kirche, Bd. 7, Freiburg: Herder, 1997, 136, 301, 302, 394.

35. Ein Studienkonzept zur Verbesserung der Ostkompetenz, in: Der Donauraum 36 (1996 [1997]) 1/2, 99-103.

36. Griechisches Schriftdenken und slavische Schriftlichkeit: Glagolica und Kyrillica zwischen Verschriftung und Verschriftlichung, in: Verschriftung und Verschriftlichung. Aspekte des Medienwechsels in verschiedenen Kulturen und Epo- 
chen, herausgegeben von Christine Ehler u. Ursula Schaefer (ScriptOralia 94), Tübingen 1998, 132-155.

37. (mit Maria Schnitter) Imena i dati - predvaritelni rezultati na izsledvaneto na $\mathrm{t}$. n. ,zapaden fond“ v slavjanskite mesecoslovi, in: Medievistika i kulturna antropologija. Sbornik v čest na 40-godišnata tvorčeska dejnost na prof. Donka Petkanova, ed. Anisava Miltenova. Sofija 1998, 27-36.

38. (mit Maria Schnitter) Kyrillomethodianische Miszellen. Westliche Einträge in den ältesten kirchenslavischen Kalendarien, in: FS für Klaus Trost zum 65. Geburtstag, herausgegeben von E Hansack, W. Koschmal, N. Nübler, R. Večerka (Die Welt der Slaven. Sammelbände/Sborniki, Bd. 5). München: O. Sagner 1999, 259-288.

39. Eine Wiener Schule der Slawistik?, in: Slawistik an der Universität Wien 18491999, unter der Redaktion von Juliane Besters-Dilger und Heinz Miklas. Wien $1999,2-13$.

40. Fragen und mögliche Antworten zu den frühesten Zeugnissen des kyrillomethodianischen Schrifttums, in: Thessaloniki - Magna Moravia. Proceedings of the International Conference Thessaloniki, 16-19 Oct. 1997. Thessaloniki: Hellenic Association for Slavic studies / SS Cyrill and Method Center for Cultural Studies, 1999, 201-215.

41. Geleitwort zu: Gerhard Podskalsky, Theologische Literatur des Mittelalters in Bulgarien und Serbien (865-1459). München: Beck, 2000 (2 S.).

42. Zur editorischen Vorbereitung des sog. Missale Sinaiticum (Sin. slav. 5/N), in: Glagolitica - Zum Ursprung der slavischen Schriftkultur (ÖAW, Phil.-hist. K1., Schriften der Balkan-Kommission, Philologische Abt. 41), Wien 2000, 117-129, XV-XVI.

43. Stand und Entwicklungsperspektiven der Slavistik in Österreich, in: Current State and Further Perspectives of Slavonic Studies in Central Europe. Proceedings of the International Workshop Prague 11-12 November 1998, ed. by Vladimír Vavř́nek. Prague: CEU/Slavonic Institute 2000, 37-40.

44. (mit J. Besters-Dilger) Geleitwort zu „150 Jahre Slawistik an der Universität Wien“, in: Wiener Slavistisches Jahrbuch 45 (1999) 2000, 7-14.

45. Ein Steckbrief zu: Anastasius Sinaita, De presbyteris peccantibus et de sacra communione, in: Godišnik na Sofijskija universitet „Sv. Kliment Ochridski“, Centăr za slavjano-vizantijski proučvanija ,Prof. Ivan Dujčev“, t. 89 (8) (2000), 73-78.

46. Bulgaristik heute und morgen, in: Bălgaristika 2001. Dokladi ot meždunarodnata rabotna srešta Sofija, 21-22 septemvri 2001g. 2. Sofija: BAN/SU „Sv. Kliment Ochridski“/Nov bălgarski universitet, 2001, 54-61.

47. Zur Einordnung des Westgutes im altkirchenslavischen Schrifttum, in: Srednovekovna christijanska Evropa: Iztok i zapad. Cennosti, tradicii, obštuvane/Medieval Christian Europe: East and West. Tradition, Values, Communications, ed. V. Gjuzelev, A. Miltenova. Sofija 2002, 114-131. 
48. Zum griechischen Anteil am glagolitischen Schriftsystem des Slavenlehrers Konstantin-Kyrill, in: Palaeoslavica 10 (2002, FS für I. Ševčenko) 1, 281-311.

49. Das Heilige als Konfliktstoff am Beispiel der ,Jugoslawien-Krise“ und ihrer Folgen, in: Ökumenisches Forum. Grazer Jahrbuch für Konkrete Ökumene 25 (2002), 97-107.

50. Die slavischen Schriften: Glagolica und Kyrillica, in: Der Turmbau zu Babel. Ursprung und Vielfalt von Sprache und Schrift. Ausstellungskatalog des Kunsthistorischen Museums, herausgegeben von W. Seipel. Bd. 3a, Wien 2003, 243249.

51. Beschreibungen von 11 glag. und kyrill. Denkmälern, in: Der Turmbau zu Babel. Ursprung und Vielfalt von Sprache und Schrift. Ausstellungskatalog des Kunsthistorischen Museums, herausgegeben von W. Seipel. Bd. 3b, Wien 2003, 236-246 (Nr. 3.5.16-26).

52. Zur Rolle der Wiener akademischen Institutionen in der Geschichte der Slavistik des 19. Jahrhunderts, in: Histoire de la slavistique. Le rôle des institutions (Travaux publiés par l'Institut d'études slaves XLIV). Sous la direction de Antonia Bernard. Paris 2003, 17-44.

53. Jesus-Abbreviatur und Verwandtes: $\mathrm{Zu}$ einigen Rätseln der glagolitischen Schriftentwicklung am Material der Azbučnaja molitva, in: Time flies. Festschrift for William R. Veder on the occasion of his departure as Professor of Slavic linguistics at the University of Amsterdam, ed. W. Honselaar, H. van der Taak et alii (Pegasus Oost-Europese Studies 2). Amsterdam 2003, 171-204.

54. Misijata na Kiril i Metodij i obedinenieto na Evropa, in: Bălgaristika/Bulgarica (Sofija) 8/2004, 9-18.

55. Misijata na Kiril i Metodij i obedinenieto na Evropa (Vortragsfassung), in: Nau$k a$ (Sofija) 4/2004, 7-12.

56. (mit Maria Djulgerova) Übersetzungs- und Originaldokumente der bulgarischen Wiedergeburt als Zeichen und Ausdruck staatlicher und zivilisatorischer Entwicklung, in: „Herrschaft“ und „Staat“. Untersuchungen zum Zivilisationswortschatz im südosteuropäischen Raum 1840-1870. Eine erste Bilanz, herausgegeben von Radoslav Katičić (ÖAW, Phil.-hist. Kl., Schriften der BalkanKommission 43). Wien 2004, 75-95.

57. Gimnografičeskie pamjatniki i razvitie drevneslavjanskich pis'mennych sistem, in: BraSlav 2 (2003) (Bratislava 2004), 54-63.

58. Analysis of Traditional Written Sources with the Aid of Modern Technologies, in: EVA 2004 Moscow Conference - 29 Nov-04 Dec 2004 (http://conf.cpic.ru /eva2004/rus/reports/report_211.html.) (7 pp.).

59. Zur Relevanz des neuen sinaitischen Materials für die Entwicklungsgeschichte der Glagolica, in: Glagoljica i hrvatski glagolizam. Zbornik radova s međunarodnoga znanstvenog skupa povodom 100. obljetnice Staroslavenske akademije i 50. obljetnice Staroslavenskog instituta (Zagreb - Krk 2.-6. listopada 2002). 
Uredili M.-A. Dürrigl, M. Mihaljević, F. Velčić. Zagreb: Staroslavenski institut/ Krčka biskupija, 2004, 389-399.

60. Zur Orientierung der Mission von Methodios und Kyrillos, in: Methodios und Kyrillos in ihrer europäischen Dimension, herausgegeben von Evangelos Konstantinou (Philhellenische Studien 10). Frankfurt a. M./Berlin etc.: Peter Lang, 2005, 53-71.

61. Kakvo ni kazva grafikata za teksta i negovija săzdatel, in: Kulturnite tekstove na minaloto: nositeli, simvoli i idei. Materiali ot Jubilejnata meždunar. naučna konferencija v čest na 60 -godišninata na prof. d.i.n. Kazimir Popkonstantinov, Veliko Tărnovo, 29-31 oktomvri 2003. Kn. I. Tekstovete na istorijata, istorija na tekstovete. Sofija: Univ. izdatelstvo „Sv. Kliment Ochridski“, 2005, 50-56.

62. (mit Stanislaus Hafner und Eleonore Ertl) Geschichte der österreichischen Slawistik, 1918-1938. Mit einem Anhang von Heinz Miklas, in: Beiträge zur Geschichte der Slawistik in den nichtslawischen Ländern, herausgegeben von Giovanna Brogi Bercoff, Pierre Gonneau, Heinz Miklas (ÖAW. Phil.-hist. Kl. Schriften der Balkan-Kommission 46 / Institut d'études slaves, Traveaux ... XLVI). Wien: ÖAW, 2005, 27-87.

63. Westkyrillisch „Đerv“ und die Herkunft der nicht-griechischen Elemente der Kyrillica, in: Johannes Reinhart/Tilman Reuther (Hrsgg.): Ethnoslavica. Festschrift für Herrn Univ.Prof. Dr. Gerhard Neweklowsky zum 65. Geburtstag. Beiträge des internationalen Symposiums des Instituts für Slawistik der Universität Klagenfurt in Zusammenarbeit mit dem Institut für Slawistik der Universität Wien, Klagenfurt, 7. bis 8. April 2006 (Wiener Slawistischer Almanach, Sonderband 65). Wien 2006, 171-178.

64. Glagolitic Alphabet in Unicode: Proposals for the Perfection of Slavonic Ranges, in: Sovremennye informacionnye technologii i pis'mennoe nasledie: ot drevnih rukopisej k èlektronnym tekstam. Materialy meždunarodnoj konferencii Iževsk, 13-17 ijulja $2006 \mathrm{~g}$. Iževsk 2006, 114-117.

65. Eine kirchenslavische Inschrift aus Thrakien, in: Bernhard Symanzik (Hrsg.), Studia Philologica Slavica. Festschrift für Gerhard Birkfellner zum 65. Geburtstag gewidmet von Freunden, Kollegen und Schülern. 2 Bde. (Münstersche Texte zur Slavistik 4). Münster 2006, 447-451.

66. (mit Iliana Čekova) „Zapadnijat fond“ svetci v cărkovnoslavjanskite Stišni sinaksari. In: Wort - Geist - Kultur. Gedenkschrift für Sergej S. Averincev, herausgegeben von J. Besters-Dilger, H. Miklas, G. Neweklowsky und F. B. Poljakov (Russkaja kul'tura v Evrope 2). Frankfurt a. M./Berlin etc.: Peter Lang, 2006, 47-125 u. 483-493 (Reg.).

67. (mit Martin Lettner, Markus Diem, Robert Sablatnig, Paul Kammerer) Registration of Multispectral Manuscript Images as Prerequisite for Computer Aided Script Description, in: Michael Grabner, Helmut Grabner (eds.), $12^{\text {th }}$ Computer Vision Winter Workshop, St. Lambrecht, Austria, February 2007, 51-58. 
68. Schrift und Bild: Zur Darstellungsfunktion der Glagolica des Slavenlehrers Konstantin-Kyrill, in: Vom Körper zur Schrift, herausgegeben von M. Schnitter, E. Vavra und H. Wenzel. Redaktion: Ch. L. Diedrichs. Sofia 2007, 45-75 u. Abb. I-IV.

69. Po sledite na Konstantinovata glagolica, in: Palaeobulgarica 31 (2007) 1, 3-26.

70. (mit Maria Djulgerova) Die Sprache als Institution einer heranwachsenden Gesellschaft: Zur Entwicklung des bulgarischen Zivilisationswortschatzes im 19. Jahrhundert, in: Gerhard Neweklowsky (Hrsg.): Herrschaft, Staat und Gesellschaft in Südosteuropa aus sprach- und kulturhistorischer Sicht. Erneuerung des Zivilisationswortschatzes im 19. Jahrhundert. Akten des Internationalen Symposiums 2.-3. März 2006 (ÖAW. Philos.-histor. Kl., Schriften der Balkan-Kommission 48). Wien 2007, 115-177.

71. Formal'naja struktura Konstantinovoj glagolicy, in: A. I. Sobolevskij i russkoe istoričeskoe jazykoznanie (k 150-letiju so dnja roždenija učenogo). Tezisy dokladov Meždunarodnoj konferencii (Moskva, 8-11 ijunja 2007g.). Moskva 2007, 43.

72. St. Catherine's Monastery on Mount Sinai and the Balkan-Slavic ManuscriptTradition. (Abridged version), in: Slovo: Towards a Digital Library of South Slavic Manuscripts (http://slovo-aso.cl.bas.bg/sinai.html). (ca. 7 S.)

73. Zum Gang der Erforschung der glagolitischen Neufunde im Katharinenkloster auf dem Sinai, in: Moskovich, Wolf/Nikolova, Svetlina (eds.): Jews and Slavs 20, Jerusalem/Sofia 2008, 48-59.

74. (mit Kazimir Popkonstantinov) Oloven amulet s glagoličeski tekst, in: Sbornikv čest na Svetlina Nikolova (Kirilo-Metodievski studii 18). Sofija 2008 - im Druck.

75. (mit Martin Lettner, Markus Diem, Robert Sablatnig) Digital Image Analysis for the Investigation of Ancient Manuscripts, in: CAAO7 - Layers of Perception, Berlin/Germany, April 2007 - im Druck.

C. Rezensionen:

1. Helmut Wilhelm ScHALlER: Bibliographie zur Balkanphilologie. Heidelberg: C. Winter, 1977. 109+2 S. In: Anzeiger für slavische Philologie 10/11 (Graz 1979), 250-256.

2. Balkan-Archiv, Neue Folge, herausgegeben von Johannes KRAMER. Bd. 1, Köln: o. Vlgsangabe. 1976, 100 S. u. 11 Ill. In: Anzeiger für slavische Philologie 10/11 (Graz 1979), 240-249.

3. Ernst HANSACK: Die Vita des Johannes Chrysostomos des Georgios von Alexandrien in kirchenslavischer Übersetzung. Bd. 1, Würzburg: JAL-Vlg., 1975, 255 S., Bd. 2: Freiburg i. Br.: U.W. Weiher, 1980, 396 S. (Monumenta linguae slavicae ... Tom. X. Tom. XIII (X, 2). In: Die Welt der Slaven XXVIII.1 (N.F. VII.1) (München 1983), 193-202. 
4. Origo characteris sclavonici. Zur altbulgarischen Literatur in Marburg (Schriften der Universitätsbibliothek Marburg 32). Marburg: Universitätsbibliothek, 1987. 192 S. In: Südosteuropa-Mitteilungen 27 (München 1987) 2/4, 289-292.

5. ECKHARDT, Thorvi: Azbuka. Versuch einer Einführung in das Studium der slawischen Paläographie. Mit einem Vorwort von Walter LEITSCH und einem bibliographisch-kritischen Nachwort von Christian HANNICK. Herausgegeben von Max Demeter Peyfuss. Wien, Köln: Böhlau, 1989 (Wiener Archiv f. Geschichte d. Slawentums u. Osteuropas 14). In: Zeitschrift für slavische Philologie 52 (Heidelberg 1991) 1, 196- 202.

6. Vladko MURDAROV, Die Wiener Slavistik und die bulgarische Sprachwissenschaft 1822-1849-1918 (Miscellanea Bulgarica 14, Wien: Verein „Freunde des Hauses Wittgenstein“, 2001), 373 S. In: Österreichische Osthefte 46 (Wien 2004), 309-312.

Heinz Miklas

Institut für Slawistik der Universität Wien

Universitätscampus AAKH, Hof 3

Spitalgasse 2, 1090 Wien, Österreich

heinz.miklas@univie.ac.at 